\title{
Rare Causes of Acute Coronary Syndrome: The JAK2 V617F Mutation-Positive Myeloproliferative Neoplasms: A Cardio-Hematological Perspective
}

\author{
Silvia Cantoni ${ }^{1}$ Claudia Colombo ${ }^{2}$ Francesco Soriano $^{3}$ Jacopo Andrea Oreglia ${ }^{3}$ Alice Sacco $^{2}$ \\ Silvio Veronese ${ }^{4}$ Dario Brunelli ${ }^{2}$ Andrea Rubboli ${ }^{5}$ Nuccia Morici ${ }^{2}$
}

${ }^{1}$ Division of Hematology, Niguarda Cancer Center, ASST Grande Ospedale Metropolitano Niguarda, Milan, Italy

2 Intensive Coronary Care Unit and De Gasperis Cardio Center, ASST Grande Ospedale Metropolitano Niguarda, Milan, Italy

${ }^{3}$ Division of Interventional Cardiology, De Gasperis Cardio Center, ASST Grande Ospedale Metropolitano Niguarda, Milan, Italy

${ }^{4}$ Division of Pathological Anatomy, ASST Grande Ospedale Metropolitano Niguarda, Milan, Italy

${ }^{5}$ Division of Cardiology, Department of Cardiovascular Diseases, AUSL Romagna, Ospedale S. Maria delle Croci, Ravenna, Italy

Thromb Haemost 2022;122:1429-1431.

Polycythemia vera (PV) and essential thrombocythemia (ET) are two myeloproliferative neoplasms (MPNs) harboring the JAK2 V617F mutation which represent an often-overlooked cause of acute coronary syndrome (ACS). However, were they promptly recognized by the cardiologist taking care of the patient, a significant improvement in long-term outcome might be obtained.

PV and ET, which share very similar clinical features and may be indistinguishable in their early phases, are characterized by inappropriately increased production of neutrophils, erythrocytes, and platelets. ${ }^{1}$

They usually run an indolent clinical course. Nevertheless, affected patients have an increased thrombotic risk: the interplay between higher levels of circulating blood cells, the endothelium, and the coagulation system leads to thrombus formation in both venous and arterial vessels. ${ }^{2-4}$

The JAK2 V617F mutation-present in over 95\% of PV and in approximately $60 \%$ of ET cases-adds significantly to this thrombotic risk by altering both the cellular and plasma compartments of hemostasis favoring a hypercoagulable state. ${ }^{2}$

Thrombotic events may precede the diagnosis of PV/ET by years and not infrequently their occurrence leads to diagnosis of either one of these disorders. ${ }^{5}$

In this setting, antiplatelet therapy alone is not adequate to prevent further thrombotic events and cytoreductive therapy with hydroxycarbamide associated with phlebotomy when appropriate is indicated to lower platelet and
Address for correspondence Nuccia Morici, MD, Dipartimento Cardiotoracovascolare, Cardiac Intensive Care Unit and De Gasperis Cardio Center, ASST Grande Ospedale Metropolitano Niguarda, Piazza Ospedale Maggiore, 3, Milan 20162, Italy

(e-mail: nuccia.morici@ospedaleniguarda.it).

leukocyte counts and to decrease blood viscosity, in order to lower the risk of recurrent thrombosis.

Since the prothrombotic milieu of PV/ET which favors ACS is peculiar, in this research letter we aimed at exploring whether differences in baseline patient characteristics and angiography findings may exist between ACS-PV/ET patients and general population (ACS-PV/ET negative).

Medical charts and coronary angiography of all 1,394 consecutive ACS patients admitted to the intensive coronary care unit (ICCU) of Niguarda Hospital between January 2014 and December 2019 were retrospectively reviewed.

Patients were grouped according to the clinical setting: (1) patients diagnosed with JAK2 V617F mutation-positive $\mathrm{PV} / \mathrm{ET}$ during the index hospitalization (ACS-PV/ET population $=26$ patients); (2) ACS patients without a diagnosis of MPN (ACS-PV/ET-negative population $=1,368$ patients).

JAK2 wild-type and V617F alleles on genomic DNA using TaqMan allelic discrimination (TaqMan Genotype Software) was used to detect the presence of V617F mutation. Screening for the JAK-2 mutation was undertaken only in patients with persisting, above-normal reference values of neutrophil and platelet counts, suggesting presence of an underlying myeloproliferative disorder, as opposed to the transient reactive increase associated with ACS. Testing did not include the other two mutations-i.e., CALR and MPL-found in MPN since they are rarer and do not seem to confer an additional higher risk of thrombosis. ${ }^{2}$ received

October 11, 2021

accepted after revision

January 14, 2022

published online

March 4, 2022 (c) 2022. Thieme. All rights reserved. Georg Thieme Verlag KG,

Rüdigerstraße 14,

70469 Stuttgart, Germany
DOI https://doi.org/ 10.1055/a-1742-0361. ISSN 0340-6245. 


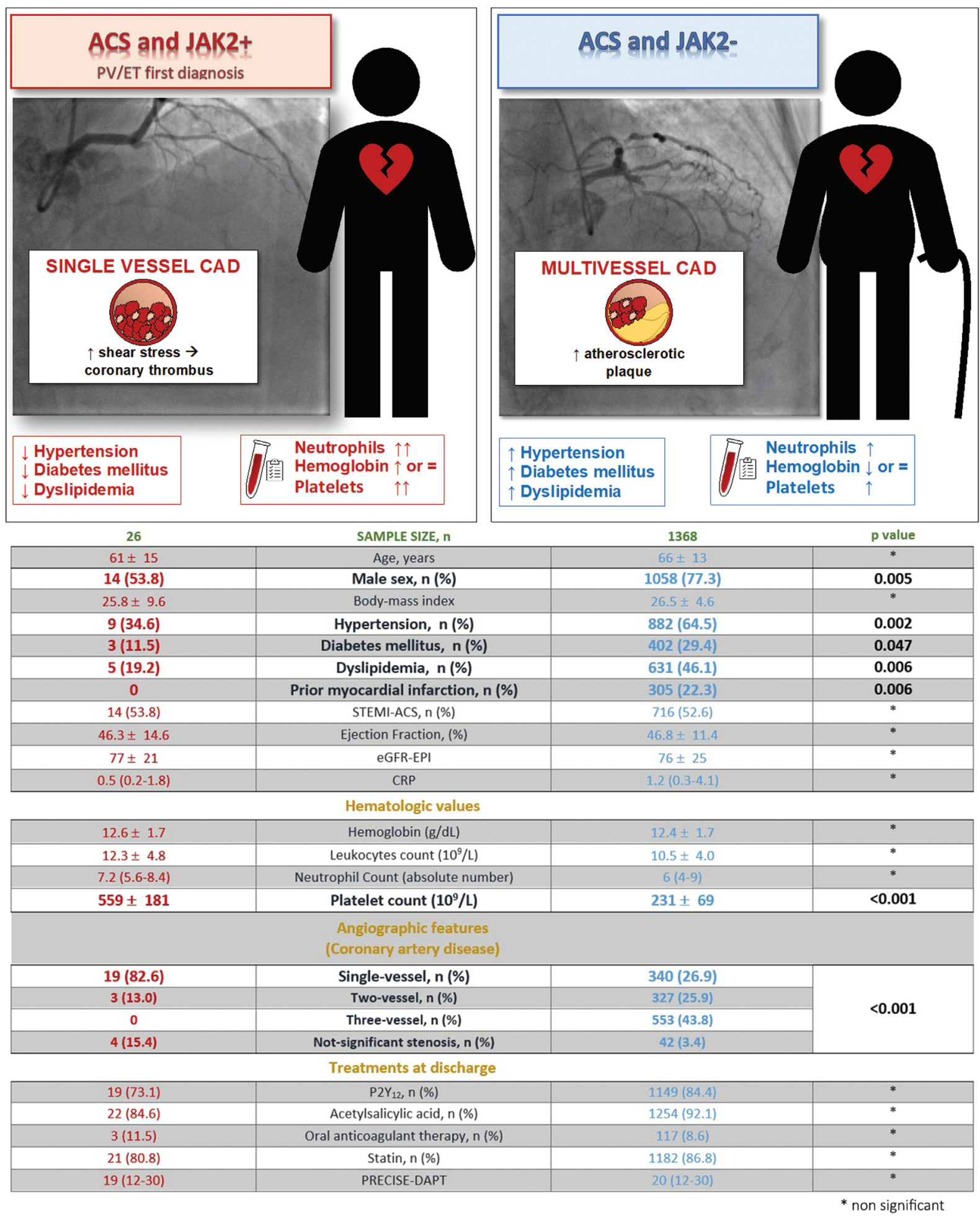

Fig. 1 Different clinical profiles between patient with acute coronary syndrome (ACS) and polycythemia vera (PV) or essential thrombocythemia (ET) compared with patients with ACS not associated with PV/ET. Statistically significant differences were reported for the following variables: gender, hypertension, diabetes mellitus, dyslipidemia, prior myocardial infarction, platelet count, coronary artery disease. Indication to oral anticoagulant was atrial fibrillation. STEMI: ST-elevation myocardial infarction.

The study was conducted in accordance with ethical principles based on the Helsinki Declaration, International Conference on Harmonization for Good Clinical Practice, and the current ethical rules. This study was approved by the local ethics committee. Patients admitted at our ICCU sign a generic data collection consent for research purpose.
Results are summarized in - Fig. 1. Notably, incidence of cardiovascular risk factors was lower and single-vessel disease was more prevalent among PV/ET patients. Two-vessel disease was also less prevalent among PV/ET patients: 13 vs. 83\%. Moreover, both single- and two-vessel diseases were also associated with high thrombotic burden (assessed by 
visual revision of angiographies) and modest atherosclerotic changes of culprit lesion as opposed to the angiography features of the control population

At discharge, all ACS-PV/ET patients received standard double antiplatelet therapy along with cytoreductive therapy in order to lower neutrophil and platelet counts, both recognized risk factors for recurrent thrombosis. Outpatient hematological follow-up was also implemented.

The composite of postdischarge overall mortality, myocardial infarction, and stroke occurred in 1 (3.8\%) patient in the ACS-PV/ET group (estimated incidence rate and lower/upper bounds of 95\% confidence intervals [CIs] [per 100 person-years] of 2.91 [0.41-20.67]) versus 104 (7.6\%) in the ACS-PV/ET-negative patients (95\% CI [per 100 personyears] $5.11[4.22-6.20])(p<0.01)$. Bleeding events $(>2$ according to Bleeding Academic Research Consortium classification) occurred only in 51 ACS-MPN-negative patients (3.7\%).

Because of the retrospective nature of our analysis, we cannot rule out with certainty that patients in the control group with early-phase PV/ET or carrying mutations other than JAK2 (i.e., CALR or MPL) might have been undiagnosed. However, PV and ET are rare disorders ${ }^{6}$ and inclusion of these potentially undiagnosed patients doubtfully would invalidate our overall results.

In PV/ET thrombotic events have been linked to quantitative (leukocytosis, thrombocytosis, elevated hematocrit) and qualitative (i.e., the JAK2 V617F mutation) abnormalities. Moreover, hyper-viscosity and the associated high shear stress in the vessel wall can lead to chronic endothelial dysfunction. This in turn activates platelets and leukocytes which are both increased in number and more "thrombogenic" as a result of procoagulant features linked to the JAK2 V617F mutation. ${ }^{2}$

Considering the anatomical sites involved (i.e., proximal segments of relatively large coronary vessels), it may be speculated that, in newly diagnosed PV/ET patients, endothelial dysfunction associated with elevated shear stress may be the main inciting factor leading to thrombus formation. In the setting of shear-induced endothelial dysfunction, the overall procoagulant features of circulating cells may give rise to thrombus formation even in the absence of underlying atherosclerotic plaques.

Isolated case reports of ACS-PV/ET patients are described in the literature with angiography findings consistent with our cases, especially mild atherosclerotic stenosis and high burden of thrombus. $^{7}$

Clinical suspicion of an underlying JAK-2-positive PV/TE stems from the peculiar angiography features (prominent single-vessel disease with high thrombotic burden) associated with neutrophilia/thrombocytosis/elevated hematocrit at admission and persisting during the index hospitalization. Neutrophilia and thrombocytosis are more reliable markers since acute drops in hemoglobin values may be observed in the setting of ACS. ${ }^{8}$

Complete blood count features need not to be overlooked and interpreted as solely reflecting a reactive change secondary to the acute ischemic event. Conversely, they should prompt hematologic evaluation aimed at establishing diagnosis and implementing a more comprehensive therapeutic strategy, including cytoreduction with hydroxycarbamide and phlebotomy.

Conflict of Interest

None declared.

\section{References}

1 Tefferi A, Barbui T. Polycythemia vera and essential thrombocythemia: 2017 update on diagnosis, risk-stratification, and management. Am J Hematol 2017;92(01):94-108

2 Barbui T, Falanga A. Molecular biomarkers of thrombosis in myeloproliferative neoplasms. Thromb Res 2016;140(Suppl 1):S71-S75

3 Carobbio A, Ferrari A, Masciulli A, Ghirardi A, Barosi G, Barbui T. Leukocytosis and thrombosis in essential thrombocythemia and polycythemia vera: a systematic review and meta-analysis. Blood Adv 2019;3(11):1729-1737

4 Guadall A, Lesteven E, Letort G, et al. Endothelial cells harbouring the JAK2V617F mutation display pro-adherent and pro-thrombotic features. Thromb Haemost 2018;118(09):1586-1599

5 Enblom A, Lindskog E, Hasselbalch $\mathrm{H}$, et al. High rate of abnormal blood values and vascular complications before diagnosis of myeloproliferative neoplasms. Eur J Intern Med 2015;26(05): 344-347

6 Nielsen C, Birgens HS, Nordestgaard BG, Bojesen SE. Diagnostic value of JAK2 V617F somatic mutation for myeloproliferative cancer in 49488 individuals from the general population. $\mathrm{Br} \mathrm{J}$ Haematol 2013;160(01):70-79

7 Xiong N, Gao W, Pan J, Luo X, Shi H, Li J. Essential thrombocythemia presenting as acute coronary syndrome: case reports and literature review. J Thromb Thrombolysis 2017;44(01):57-62

8 Leonardi S, Gragnano F, Carrara G, et al. Prognostic implications of declining hemoglobin content in patients hospitalized with acute coronary syndromes. J Am Coll Cardiol 2021;77(04):375-388 